\title{
Effects of secular evolution on the star formation history of galaxies
}

\author{
M. Fernández Lorenzo ${ }^{1}$, J. Sulentic ${ }^{1}$, L. Verdes-Montenegro ${ }^{1}$, \\ M. Argudo-Fernández ${ }^{1}$, J. E. Ruiz ${ }^{1}$, J. Sabater ${ }^{2}$ \\ and S. Sánchez-Expósito ${ }^{1}$ \\ ${ }^{1}$ Instituto de Astrofísica de Andalucía, Granada, IAA-CSIC, Granada, Spain, \\ email: mirian@iaa.es \\ ${ }^{2}$ Institute for Astronomy, University of Edinburgh, Edinburgh, UK
}

\begin{abstract}
We report the study performed as part of the AMIGA (Analysis of the interstellar Medium of Isolated GAlaxies; http://www.amiga.iaa.es) project, focused on the SDSS $(g-r)$ colors of the sample. Assuming that color is an indicator of star formation history, this work better records the signature of passive star formation via pure secular evolution. Median values for each morphological type in AMIGA were compared with equivalent measures for galaxies in denser environments. We found a tendency for AMIGA spiral galaxies to be redder than galaxies in close pairs, but no clear difference when we compare with galaxies in other (e.g. group) environments. The $(g-r)$ color of isolated galaxies presents a Gaussian distribution, as indicative of pure secular evolution, and a smaller median absolute deviation (almost half) compared to both wide and close pairs. This redder color and lower color dispersion of AMIGA spirals compared with close pairs is likely due to a more passive star formation in very isolated galaxies. In Fig. 1, we represent the size versus stellar mass for early and late-type galaxies of our sample, compared with the local relations of Shen et al. (2003). The late-type isolated galaxies are $\sim 1.2$ times larger or have less stellar mass than local spirals in other environments. The latter would be in agreement with the passive star formation found in the previous part.

We acknowledge Grant AYA2011-30491-C02-01, P08-FQM-4205 and TIC-114.
\end{abstract}

Keywords. galaxies: general, galaxies: fundamental parameters, galaxies: evolution

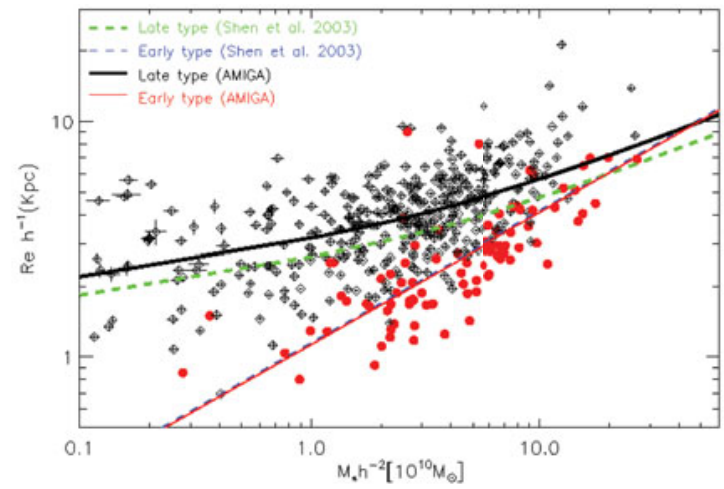

Figure 1. Stellar mass size relation for the AMIGA and Shen et al. (2003) samples. Open diamonds are the AMIGA late-types and solid points are the AMIGA early-types.

\section{References}

Shen, S., Mo, H. J., White, S. D. M., et al. 2003, MNRAS, 343, 978 